\title{
Evaluation of Left Ventricular Function by Three-Dimensional Speckle-Tracking Echocardiography in Patients with Chronic Kidney Failure
}

\author{
Yubo Wang ${ }^{1}$, Hui Huang ${ }^{1}$, Shan Lin $^{1}$, Meijia Hao ${ }^{1}$, Lujiao He ${ }^{1}$, Jun Wen ${ }^{1}$, Yahui Weng ${ }^{1}$, \\ Jiangyi Wang ${ }^{1}$, Kun $\mathrm{Liu}^{1}$, and Xiaojun $\mathrm{Bi}^{1}$ \\ ${ }^{1}$ Affiliation not available
}

October 20, 2020

\begin{abstract}
Objective To establish a quantitative evaluation of the left ventricle's systolic function in patients with chronic kidney failure (CKF) by three-dimensional speckle-tracking echocardiography(3D-STE). Methods Two-dimensional transthoracic echocardiography and three-dimensional transthoracic echocardiography were performed on 30 patients with CKF. The ejection fraction (EF), mass and global peak longitudinal strain (GL), global circumferential strain (GC), global area strain (GA), and global radial strain (GR) of the left ventricle were calculated. Results (1) EF, GL, GC, GA, and GR in the CKF group were significantly lower than in the control group. Meanwhile, GL, GC, GA, and GR were well correlated with EF. (2) For patients with normal EF in the CKF group, GL, GC, GA, and GR were lower than in the control group, while the left ventricular mass was significantly higher than in the control group. (3) For patients with hypertension in the CKF group, EF, GL, GC, GA, and GR calculated using three-dimensional echocardiography were significantly lower than in patients with normal blood pressure; however, the myocardial mass was higher. Conclusions Parameters calculated using three-dimensional speckle-tracking software were lower in the CKF group. Simultaneously, the left ventricular mass was higher than in the control group, showing that the myocardial contraction function was impaired and that myocardial reconstruction occurred.
\end{abstract}

\section{Introduction}

Severe cardiovascular complications often occur in patients with chronic kidney failure (CKF). About half of end-stage renal patients with the disease die of cardiovascular disease. The mortality of dialysis patients is 10-20 times higher than that of healthy people, and the critical factors are ischemic cardiomyopathy and myocardial hypertrophy. It is crucial to find the cardiac function changes of patients affected with chronic renal failure.

Traditional echocardiography, such as two-dimensional speckle-tracking echocardiography can assess the changes in the heart's structure and function in renal failure patients. It can perform semi-quantitative measurements of the heart's deformation ${ }^{[1]}$. However, two-dimensional methods have caveats; notably, they cannot display the three-dimensional motion of the heart itself. Although the left ventricular ejection fraction $(\mathrm{EF})$ is normal in CKF patients, traditional ultrasound examination cannot detect abnormal cardiac function sensitively.

Three-dimensional speckle-tracking echocardiography can accurately calculate the deformation and motion of the heart in renal failure patients and evaluate the integral and local left ventricular features by the sequence tracing movement route of myocardial echo spots at different interframes in three-dimensional space.

This study assessed the cardiac motion of CKF patients in three-dimensional space using three-dimensional speckle-tracking technology to accurately detect abnormal cardiac function in renal failure patients. 


\section{Research objects}

Thirty patients with CKF were selected in our hospital from May 2012 to July 2012. Among the selected cases, 25 were male, ten were female, and the mean age was $44 \pm 13$ years. In the normal control group, 12 cases were male, seven were female, and the mean age was $44 \pm 14$ years.

\section{Instruments and methods}

\section{Instruments}

Informed consent was obtained from patients before they were enrolled in the study. Vivid E9 ultrasonic echocardiography was supplied by GE using $4 \mathrm{~V}$ and M5S probe, frequency from 1.7 to $3.4 \mathrm{MHz}$, mechanical index of 1.1. Imaging acquisition: The subjects were told to breathe calmly and to relax. Blood pressure was measured three times before checking. Creatinine, urea, and uric acid levels were recorded. Upon connecting the ECG, patients were told to adopt a left lateral position and breathe quietly. Images were stored by an M5S probe placed near the left side of the sternum for the shaft section and apex four-chamber. The full volume 4v mode was selected, and the depth and full volume fan Angle were adjusted. A sound beam covered the left ventricle. Frame frequency stayed above 25 frames per second. After telling patients to breathe calmly, images of the cardiac cycle were selected and stored on the hard disk for offline analysis.

\section{Image analysis}

Images of the left ventricle were taken. The left interior and interventricular diameters were measured beside the parasternal left ventricle at a long axis section. EF was measured from the apical four-chamber view. After entering the Echopac workstation and enabling $4 \mathrm{v}$ analysis, the apical four-chamber, apical two-chamber, and left apical ventricle at a long axis section were displayed according to the order on the screen. Three short-axis images of the mitral valve, papillary muscle, and apex level were taken. The Angle of each section was adjusted to display two-dimensional structures of the left ventricular heart. From the endocardial and epicardial views, a point was selected so that the system drew images automatically at the heart valve ring and apex when diastolic and systolic readings ended. Each section had to be adjusted to fit the endocardial and epicardial surfaces. The computer automatically calculated the heart EF, left ventricular weight, global longitudinal strain, global circumferential strain (GC), global area strain (GA), and global radial strain (GR). The first manipulator measured above parameters twice repeatedly, and then another manipulator measured the parameters a third time, resulting in the final average.

\section{Statistical analysis}

SPSS 20.0 software was used. All measurement data were presented as mean \pm standard deviation (SD). P $<0.05$ was considered statistically significant. The unpaired t-test was used to compare the group of CKF patients with the control group. The Pearson correlation test was used to compare the left ventricular EF, GL, GC, GR, GA, and left ventricular weight between groups. To assess intraobserver and interobserver variability, 30 random subjects (15 from each group) were analyzed on two separate occasions by the same operator and two different operators, respectively. Intraclass correlation coefficients (ICC) were assessed using a two-way mixed-effects model for intraobserver variability and a two-way random-effects model for interobserver variability. ICCs $>0.80$ were considered excellent and 0.60 to 0.80 good.

\section{Results}

A decreased EF was observed in CKF patients by three-dimensional speckle-tracking software. The GL, GC, GA, GR levels were also decreased compared to healthy controls (figures 1 and 2). The difference was considered statistically significant (table 1). EF by three-dimensional speckle-tracking software is correlated with GL, GC, GA, GR, and the correlation coefficients are 0.741, 0.925, 0.874, and 0.882, respectively. The measured values of the left ventricular EF, GL, GC, GR, GA, and left ventricular weight were highly consistent.

(1) Parameters calculated in the CKF group using three-dimensional speckle-tracking software compared with healthy controls. 
(2) GL, GC, GA, and GR levels decreased compared to the healthy controls, the measurement of EF is normal in CKF patients, and the left ventricular mass was significantly higher in controls compared to the controls (Table 2).

(3) For CKF patients with hypertension, EF, GL, GC, GA, and GR, calculated using three-dimensional echocardiography, were significantly lower than those with normal blood pressure, and myocardial mass was lower too (table 3$)$.

(4) Intra- and Interobserver Variability

For intraobserver variability, ICCs were considered excellent for the vast majority of deformation parameters. For interobserver variability, ICCs were considered excellent or good (Table 4).

\section{Discussion}

Many CKF patients have suffered cardiovascular disease, hypertension, and diabetes before hemodialysis. Such risk factors can accelerate vascular diseases and are linked to higher ventricular hypertrophy, myocardial fibrosis, valvular disease, arrhythmia, and sudden death ${ }^{[2]}$. Meanwhile, CKF patients exhibit increased risk factors related to the disease itself, including metabolic waste in the heart's deposition, enhanced oxidative stress, inflammation, blood volume, and overload pressure ${ }^{[3]}$. In CKF patients undergoing hemodialysis, coronary artery stenosis, coronary reserve, destruction of microcirculation, and an overload of pressure and capacity causes cardiac remodeling and function change, which damages the myocardial structure. Long-term overload of the left ventricle leads to myocardial cell death and fibrosis, destruction of ventricular diastolic, the discordance of ventricular electrical conduction, heart cavity expansion, and compensatory hypertrophy $[4,5]$. Thus, despite hemodialysis, all CKF patients display abnormalities in cardiac function, and a good assessment would guide clinical decisions.

Speckle-tracking is an echocardiographic method based on tracking characteristic speckle patterns created by interfering with ultrasound beams in the myocardium, which provides a good method for the study of myocardial mechanics ${ }^{[6]}$. Studies have used two-dimensional speckle-tracking technology to evaluate cardiac function in CKD patients. They found that risk factors such as GC, GR, and reduction of radial strain were associated with coronary artery disease closely. Using logistic regression analysis, they found that segments of radial strain lower than six or more are strongly associated with coronary artery disease ${ }^{[7]}$. Longitudinal strain can detect abnormal cardiac contraction under normal $\mathrm{EF}^{[8]}$. Although many studies have shown that two-dimensional speckle-tracking technology could detect changes in myocardial contraction with ischemic cardiomyopathy early ${ }^{[9]}$, these studies were limited to a single plane. Therefore, the cardiac motion would remove the plane of speckle-tracking, and motion tracking in that plane was lost. Three-dimensional speckletracking technology overcame this issue ${ }^{[10,11]}$. Thus, it can evaluate the myocardial motion more effectively and become more popular among clinicians ${ }^{[12-14]}$.

In this study, we found that the EF of left ventricular function, GL, GC, GA, and GR showed decreased levels compared to patients in the normal control group. Thus, the cardiac function of CKF patients with risk factors was impaired. Cardiac function was accurately evaluated using three-dimensional speckle-tracking technology, and $\mathrm{EF}$ of left ventricular had a good correlation and repeatability, in which the GC and EF had a better correlation ( $\mathrm{r}=0.925)$. GL, GA, and GR showed decreased levels compared with healthy controls when the EF of patients with CKF was normal. The myocardial weight of the left ventricle was significantly higher than in the control group. However, the EF of the left ventricle was normal, and the myocardial contraction function was impaired. Myocardial reconstruction had occurred. GC was not considered statistically significant in four strain-related parameters. The myocardial surface was in the normal range in the strain of the circumferential direction. The shortage could be due to the small sample used in this study. GL, GA, and GR were more sensitive than GC when the myocardial function was impaired in the compensatory stage. We provided a better method to evaluate impaired myocardial functions in CKF patients.

During CKF, the clinical and biochemical results were not statistically significant for differentiating CKF patients with hypertension from CFK patients without hypertension. However, using three-dimensional 
speckle-tracking technology, we found that EF, GL, GC, GA, and GR in CKF patients with hypertension were significantly lower than in CFK patients without hypertension. Furthermore, the left ventricular myocardial weight was significantly higher in CFK patients with hypertension compared to CKF patients without hypertension. Thus, hypertension could aggravate myocardial impairment and lead to myocardial reconstruction. We found that, unlike the clinical and biochemical results, three-dimensional speckle-tracking technology could detect myocardial impairment. Thus, our method is suitable for clinicians.

This study did not analyze heart changes before and after dialysis in CKF patients. Because of this, we only obtained a few troponin values of cases with myocardial impairment. Most cases did not display the result that is the gold standard for myocardial impairment.

\section{Conclusion}

We found that myocardial impairments and reconstruction had occurred in CKF patients. In addition, hypertension could aggravate myocardial impairment. Three-dimensional speckle-tracking technology can quantitatively evaluate cardiac function in CKF patients early and had good repeatability. Thus, we present an enhanced method that can evaluate cardiac function in CKF patients.

\section{Acknowledgements}

Not applicable.

\section{Author's contributions}

KL, YBW: Data collection and analysis, manuscript writing. XBB: Data collection, project development, manuscript editing. HH, SL, MJH, LJH, JW, YHW, JYW: Data collection, manuscript editing. All authors read and approved the final manuscript.

\section{References}

[1] Perk G, Kronzon I. Non-Doppler two-dimensional strain imaging for evaluation of coronary artery disease. Echocardiography 2009, 26: 299.

[2] Pecoits-Filho R,Barberato,SH, et al.Echocardiography in chronic kidney disease:diagnostic and prognostic implications.Nephron Clin Pract 2010, 114: 242-247.

[3] Glassock RJ, Pecoits-Filho R, Barberato SH, et al. Left ventricular mass in chronic kidney disease and ESRD. Clin J Am Soc Nephrol 2009, 4: 679-687.

[4] Parfrey PS,Foley RN.The clinical epidemiology of cardiac disease in chronic renal failure.J Am Soc Nephrol 1999, 10: 1606-1615.

[5] Peng T, Li X, Hu Z, et al. Predictive role of endothelin in left ventricular remodeling of chronic kidney disease. Renal Failure 2018, 40(1):183-188.

[6] Amundsen BH, Helle-Valle T, Edvardsen T, et al. Noninvasive myocardial strain measurement by speckle tracking echocardiography: Validation against sonomicrometry and tagged magnetic resonance imaging. J Am Coll Cardiol 2006, 47: 789-793.

[7] Liu YW, Su CT, Wang SP, Yang CS, et al. Application of speckle-tracking echocardiography in detecting coronary artery disease in patients with maintenance hemodialysis. Blood Purif 2011, 32: 38-46.

[8] Liu YW, Tsai WC, Su CT, et al. Evidence of left ventricular systolic dysfunction detected by automated function imaging in patients with heart failure and preserved left ventricular ejection fraction. J Card Fail 2009, 15: 782-786.

[9] Tsai WC, Liu YW, Huang YY, et al. Diagnostic value of segmental longitudinal strain by automated function imaging in coronary artery disease without left ventricular dysfunction. J Am Soc Echocardiogr 2010, 23: 1183-1202. 
[10] Perez de Isla L, Balcones DV, Fernandez-Golfin C, et al.Three-dimensional-wall motion tracking: A new and faster tool for myocardial strain assessment: Comparison with two-dimensional-wall motion tracking. J Am Soc Echocardiogr 2009, 22: 325-330.

[11] Saito K, O kura H, Watanabe N, et al. Comprehensive evaluation of left ventricular strain using speckle tracking echocardiography in normal adults: Comparison of three-dimensional and two-dimensional approaches. J Am Soc E chocardiogr 2009, 22: 1025-1030.

[12] Baccouche H, Maunz M, Beck T, et al. Echocardiographic assessment and monitoring of the clinical course in a patient with Tako-Tsubo cardiomyopathy by a novel 3D-speckle-tracking-strain analysis. Eur J Echocardiogr 2009, 10: 729-734.

[13] Kleijn SA, Brouwer WP, Aly MF, et al. Comparison between three-dimensional speckle-tracking echocardiography and cardiac magnetic resonance imaging for quantification of left ventricular volumes and function. Eur Heart J Cardiovasc Imaging 2012, 13: 834-839.

[14] Nesser HJ, Mor-Avi V, Gorissen W, et al. Quantification of left ventricular volumes using threedimensional echocardiographic speckle tracking: comparison with MRI. Eur Heart J 2009, 30: 1565-1571.

Table 1: The measurement of several parameters in CKF patients compared to healthy controls.

\begin{tabular}{llll}
\hline Parameters & CKF group (30 cases) & Control group (25 cases) & $P$ \\
\hline EF (\%) & $48.07 \pm 12.73$ & $59.00 \pm 6.75$ & 0.0004 \\
GL (\%) & $12.43 \pm 3.42$ & $18.80 \pm 3.08$ & $<0.0001$ \\
GC (\%) & $13.07 \pm 3.40$ & $16.80 \pm 3.34$ & 0.0002 \\
GA (\%) & $22.73 \pm 5.63$ & $31.16 \pm 4.32$ & $<0.0001$ \\
GR (\%) & $35.90 \pm 11.25$ & $50.64 \pm 10.46$ & $<0.0001$ \\
\hline
\end{tabular}

EF, ejection fraction; GL, global peak longitudinal strain; GC, global circumferential strain; GA, global area strain; GR, global radial strain. Data are expressed as mean $\pm \mathrm{SD}$.

Table 2: The measurement of EF is normal in CKF patients compared to healthy controls.

\begin{tabular}{llll}
\hline Parameters & \multicolumn{2}{l}{ CKF with normal EF (19 } & \\
cases) & Controls group (23 cases) & $P$ \\
\hline EF (\%) & $56.26 \pm 5.26$ & $57.65 \pm 5.37$ & 0.4160 \\
GL (\%) & $14.26 \pm 1.86$ & $18.48 \pm 2.98$ & $<0.001$ \\
GC (\%) & $14.11 \pm 1.68$ & $16.43 \pm 3.29$ & 0.0094 \\
GA (\%) & $25.95 \pm 2.72$ & $30.65 \pm 4.19$ & 0.0002 \\
GR (\%) & $42.79 \pm 5.66$ & $49.22 \pm 9.81$ & 0.0179 \\
ED Mass (g) & $179.74 \pm 61.09$ & $119.04 \pm 20.47$ & $<0.001$ \\
ES Mass (g) & $165.47 \pm 48.48$ & $112.91 \pm 18.32$ & $<0.001$ \\
\hline
\end{tabular}

EF, ejection fraction; GL, global peak longitudinal strain; GC, global circumferential strain; GA, global area strain; GR, global radial stain; ED, end-diastole; ES, end-systole. Data are expressed as mean \pm SD.

Table 3: Parameters calculated in the CKF group with normal blood pressure by three-dimensional speckletracking software compared with hypertension in the CKF group.

\begin{tabular}{llll}
\hline Parameters & CKF with normal blood pressure (16 cases) & Hypertension in the CKF group (14 cases) & 1 \\
\hline EF $(\%)$ & $56.88 \pm 5.38$ & $38.00 \pm 11.14$ & $0.36 \pm 3.52$ \\
GL $(\%)$ & $14.25 \pm 2.02$ & 10.5
\end{tabular}




\begin{tabular}{llll}
\hline Parameters & CKF with normal blood pressure (16 cases) & Hypertension in the CKF group (14 cases) & \\
\hline GC (\%) & $15.31 \pm 1.72$ & $10.50 \pm 2.99$ & \\
GA (\%) & $26.00 \pm 2.92$ & $19.00 \pm 5.66$ & \\
GR (\%) & $43.63 \pm 5.73$ & $27.07 \pm 9.35$ & 0 \\
ED Mass (g) & $201.09 \pm 60.82$ & $112.6 \pm 31.06$ & 0 \\
ES Mass (g) & $182.00 \pm 36.97$ & $642.31 \pm 357.20$ & 0 \\
Cr (umol/L) & $598.09 \pm 432.98$ & $477.43 \pm 155.80$ & 0 \\
UA (umol/L) & $433.18 \pm 186.25$ & $27.34 \pm 19.47$ & 0 \\
Urea (mmol/L) & $28.73 \pm 11.85$ & $46.46 \pm 39.21$ & 0 \\
Time of the disease (mouth) & $58.32 \pm 50.47$ & & \\
\hline
\end{tabular}

$\mathrm{EF}$, ejection fraction; GL, global peak longitudinal strain; GC, global circumferential strain; GA, global area strain; GR, global radial stain; ED, end-diastole; ES, end-systole; Cr, creatinine; UA, uric acid. Data are expressed as mean $\pm \mathrm{SD}$.

Table 4 Intraobserver and interobserver ICCs for parameters of left ventricular EF, GL, GC, GR, GA, and ES.

\begin{tabular}{lll}
\hline & Intraobserver & Interobserver \\
EF & 0.884 & 0.825 \\
GL & 0.923 & 0.896 \\
GC & 0.857 & 0.792 \\
GA & 0.897 & 0.834 \\
GR & 0.935 & 0.901 \\
ED Mass & 0.882 & 0.836 \\
ES Mass & 0.846 & 0.855 \\
\hline
\end{tabular}

EF, ejection fraction; GL, global peak longitudinal strain; GC, global circumferential strain; GA, global area strain; GR, global radial stain; ED, end-diastole; ES, end-systole.

\section{Figure Legends}

Figure 1: 3D offline analysis of the control group. (A) LV global longitudinal strain was -18\%; (B) LV global circumferential strain was -18\%; (C) LV global radial strain was -54\%; (D) LV global area strain was $-33 \%$.

Figure 2: 3D offline analysis of the CKF group. (A) LV global longitudinal strain was -8\%; (B) LV global circumferential strain was -7\%; (C) LV global radial strain was -18\%; (D) LV global area strain was -14\%. 

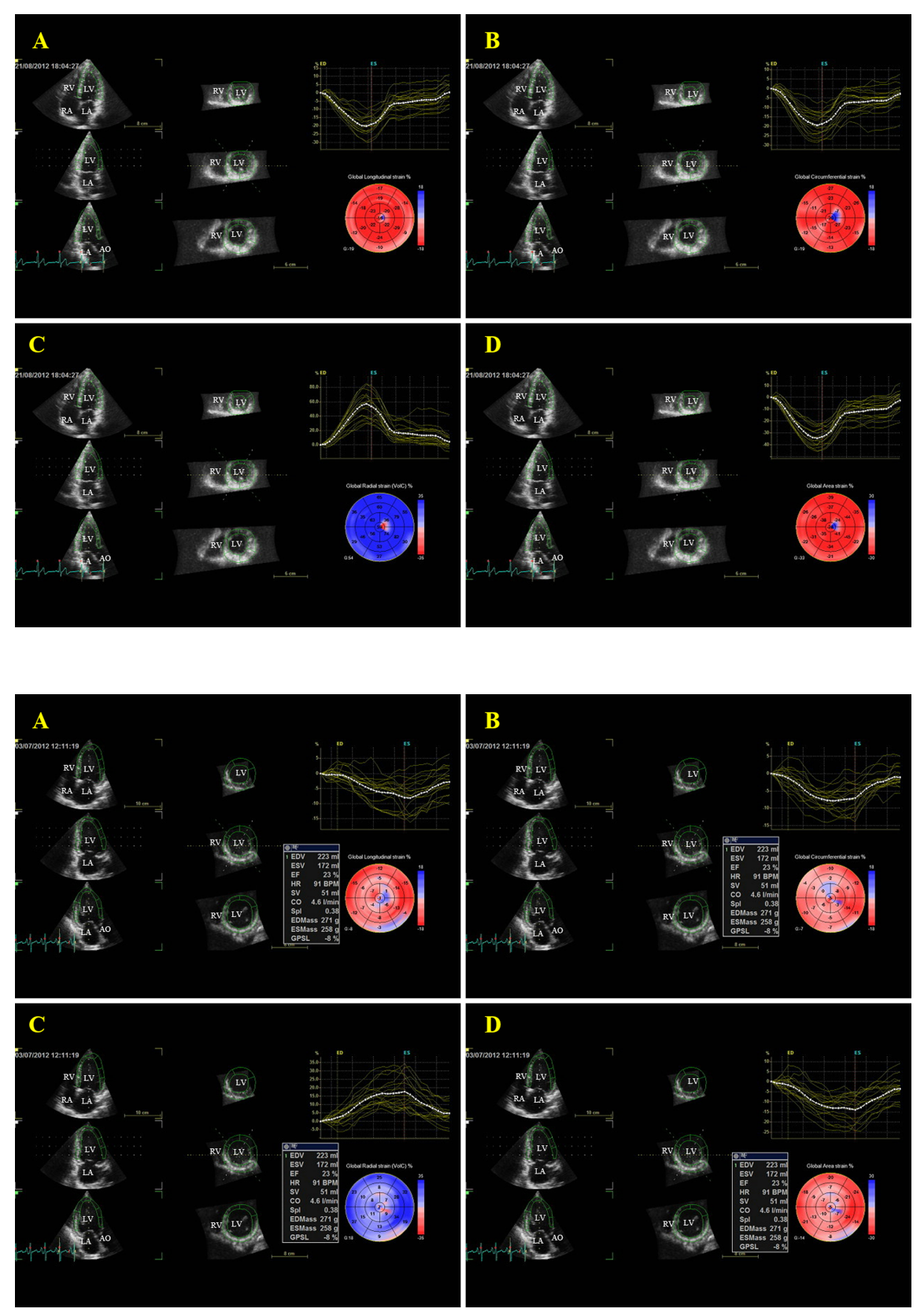